October 31, 1999

rev. 21. 1. 2000

\title{
Polyakov Loop Percolation and Deconfinement in SU(2) Gauge Theory
}

\author{
Santo Fortunato and Helmut Satz \\ Fakultät für Physik, Universität Bielefeld \\ D-33501 Bielefeld, Germany
}

\begin{abstract}
:
The deconfinement transition in $S U(2)$ gauge theory and the magnetization transition in the Ising model belong to the same universality class. The critical behaviour of the Ising model can be characterized either as spontaneous breaking of the $Z_{2}$ symmetry of spin states or as percolation of appropriately defined spin clusters. We show that deconfinement in $S U(2)$ gauge theory can be specified as percolation of Polyakov loop clusters with Fortuin-Kasteleyn bond weights, leading to the same (Onsager) critical exponents as the conventional order-disorder description based on the Polykov loop expectation value.
\end{abstract}

Colour deconfinement is a well-defined phase transition in finite temperature $S U(2)$ gauge theory; the expectation value of the Polyakov loop serves as an order parameter determining the onset of a spontaneous breaking of a global $Z_{2}$ symmetry [1, 2]. The resulting critical behaviour belongs to the universality class of the Ising model, conjectured on the basis of effective theories [3, 国 and confirmed by lattice studies [5]. In full QCD, dynamical quarks act as (small) external field; hence the conventional formalism of spontaneous symmetry breaking does not define an order parameter, and it is not clear if deconfinement remains a genuine phase transition. It therefore seems helpful to consider an alternative approach to critical behaviour in the Ising model, which may be more readily generalizable to full QCD.

The magnetization transition in the Ising model (in the absence of an external magnetic field) can be described either as the spontaneous breaking of the $Z_{2}$ symmetry of the theory by spin states or as percolation of cluster states appropriately defined in terms of the basic spin-spin interaction [6]. More specifically, the partition function on a twodimensional lattice of $L^{2}$ sites is conventionally defined as sum over all possible spin states $\{s\} \equiv\left\{s_{i}= \pm 1 \forall i=1, \ldots, L^{2}\right\}$,

$$
Z(T)=\sum_{\{s\}} b(\{s\})
$$

with the Boltzmann weight

$$
b=\exp \left\{(J / T) \sum_{\langle i, j\rangle} s_{i} s_{j}\right\},
$$


where $T$ is the temperature and $J$ the spin-spin coupling strength; the sum $\langle i, j\rangle$ runs over next neighbour spin pairs, with $i<j$. Equivalently, it can be written [7] as sum over all $2^{E}$ clusters states $G$, specified in terms of $l$ bonds forming $C$ connected clusters on a lattice consisting of $E$ links,

$$
Z(T)=\sum_{G} 2^{C} v^{l}
$$

where

$$
v=(\exp \{2(J / T)\}-1)
$$

defines the bond weight. Note that Eqs. (11) and (3) differ by a T-dependent factor related to the zero in energy.

In the spin formulation, the order parameter is given by the spontaneous magnetization $m(T)$, defined as the average spin per lattice site,

$$
m(T)=\langle|s|\rangle \equiv \frac{\sum_{\{s\}}\left\{\left|\sum_{i} s_{i}\right| / L^{2}\right\} b(\{s\})}{\sum_{\{s\}} b(\{s\})} .
$$

Its behaviour near the critical point is governed by the critical exponent $\beta$, with

$$
m(T) \sim\left(T_{c}-T\right)^{\beta}, \quad T \lesssim T_{c}
$$

the divergence of the corresponding susceptibility is with

$$
\chi_{m}(T) \sim\left\langle s^{2}\right\rangle-\langle|s|\rangle^{2} \sim\left|T-T_{c}\right|^{-\gamma}
$$

determined by the exponent $\gamma$.

In the percolation formulation, the order parameter becomes the percolation strength $P(T)$, defined as the probability that a randomly chosen site in the thermodynamic limit belongs to an infinite cluster,

$$
P(T) \sim\left(T_{c}-T\right)^{\beta}, \quad T \lesssim T_{c}
$$

The corresponding susceptibility is the average cluster size $S(T)$, excluding percolating clusters, and its divergence is governed by

$$
S(T) \sim\left|T-T_{c}\right|^{-\gamma}
$$

in the vicinity of the critical point.

Spin and cluster formulations thus provide two equivalent ways to specify the critical behaviour of the Ising model. While the spin version is based on the onset of spontaneous breaking of the global $Z_{2}$ symmetry of the Ising Hamiltonian, the cluster version uses the onset of percolation of clusters whose Fortuin-Kasteleyn bond weights are also determined by Ising dynamics.

Since for a fixed space dimension $d$, the critical behaviour of the Ising model and of finite temperature $S U(2)$ gauge theory are in the same universality class, one may expect that deconfinement can also be formulated as percolation. The aim of this paper is to show that for $d=2$ and for a specific lattice regularization, this is indeed the case. The generalization to $d=3$ appears straight-forward and is in progress. 
To implement the cluster formulation, we use the droplet approach introduced by Coniglio and Klein [8]. For the Ising model, this method generates equilibrium configurations using the Boltzmann weights and then defines clusters as regions of parallel spins connected by bonds, using Fortuin-Kasteleyn bond weights

$$
p=1-\exp \{-2(J / T)\} .
$$

The use of both Boltzmann and bond weights can be avoided, and there exist implementations using the Fortuin-Kasteleyn bond weights only [10 - 11]. In our context, the Coniglio-Klein implementation seems preferable mainly for computational reasons.

In the Ising model, clusters are thus defined as regions of parallel spins connected by bonds, with the probability for bonding given by Eq. (10). In two-dimensional $S U(2)$ lattice gauge theory, the underlying manifold becomes a $N_{s}^{2} \times N_{t}$ lattice, with $N_{s}$ sites in each spatial and $N_{t}$ sites in the temperature direction. The Ising spins $s_{i}= \pm 1$ at sites $i=1, \ldots, L^{2}$ are replaced by Polyakov loops $L_{i}$,

$$
L_{i} \sim\left\{\operatorname{Tr} \prod_{\tau=1}^{N_{t}} U_{(i ; \tau, \tau+1)}\right\},
$$

where $U_{(i ; \tau, \tau+1)}$ are $S U(2)$ matrices associated to the link at spatial site $i$ connecting the temporal planes $\tau$ and $\tau+1$.

The matrix product in Eq. (11) becomes a loop closed by periodicity in the temperature direction. We thus replace the discrete spin values $s_{i}= \pm 1$ by spins of continuous size $L_{i}= \pm\left|L_{i}\right|$ at each lattice site $i$. Clusters are now defined as regions of like-sign Polyakov loops (say $L_{i} \geq 0$ ) connected by bonds distributed according to the bond weight

$$
p_{i, j}=1-\exp \left\{-2 \kappa L_{i} L_{j}\right\},
$$

where $\kappa$ is determined by the dynamics of $S U(2)$ gauge theory; it corresponds to the $J / T$ in the Ising weight Eq. (??).

The identification of the underlying dynamics as $S U(2)$ gauge theory is contained in the $\kappa$ in Eq. (12). A general solution for this does not seem to exist so far. In the context of universality studies [3, 国, the $S U(2)$ action is formally reduced to an effective action in terms of the Polyakov loop variables $L_{i}$, so it should be possible to identify $\kappa$ in such a framework. We shall here make use of strong coupling calculations [12], in which

$$
\kappa \simeq(\bar{\beta} / 4)^{2}
$$

was found to provide a good approximation (90\% accuracy) for $N_{t}=2$; here $\bar{\beta}=\left(4 / g^{2}\right)$ denotes the coupling parameter in the $S U(2)$ lattice action.

With this, the Polyakov loop percolation problem is fully defined, and we proceed with a lattice study of the percolation strength $P(\bar{\beta})$ and the cluster size $S(\bar{\beta})$. Our analysis is based on four sets of data on $N_{s}^{2} \times 2$ lattices, with $N_{s}=64,96,128$ and 160 . The simulations were carried out on workstations for $N_{s} \leq 128$ and on a Cray T3E (ZAM, Jülich) for $N_{s}=160$. The updates consist of one heatbath and two overrelaxation steps. For the $64^{2} \times 2$ and $96^{2} \times 2$ lattices we evaluated configurations every six updates, for $128^{2} \times 2$ and $160^{2} \times 2$ every eight updates, measuring in each case $P(\bar{\beta})$ and $S(\bar{\beta})$. The 


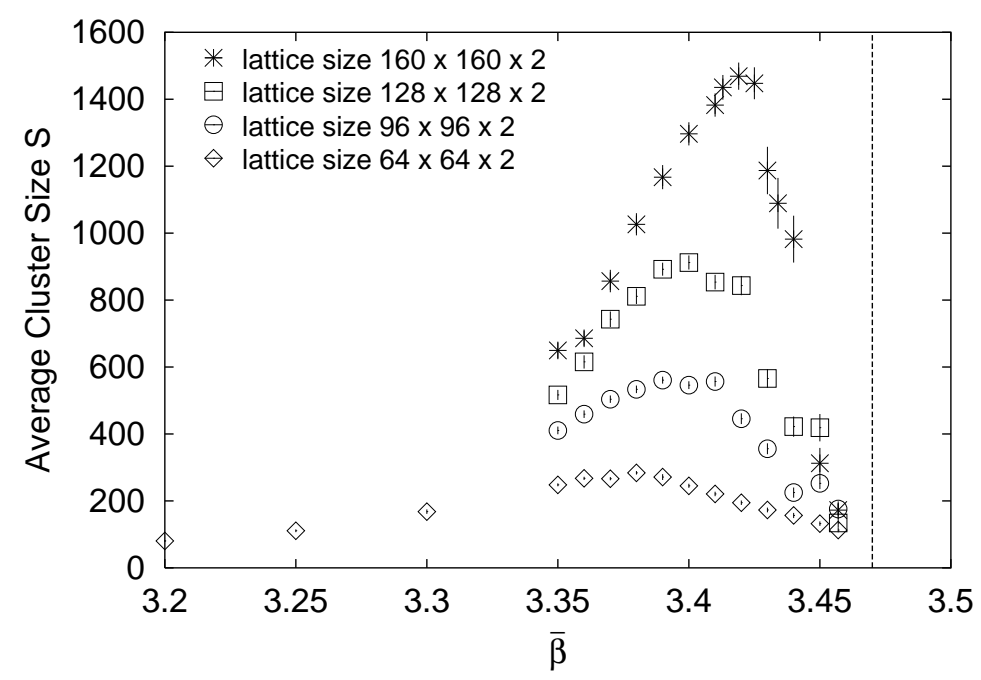

Figure 1: Cluster size as function of $\bar{\beta}$ near the critical point $\bar{\beta}_{c}(L)$ (dashed line).

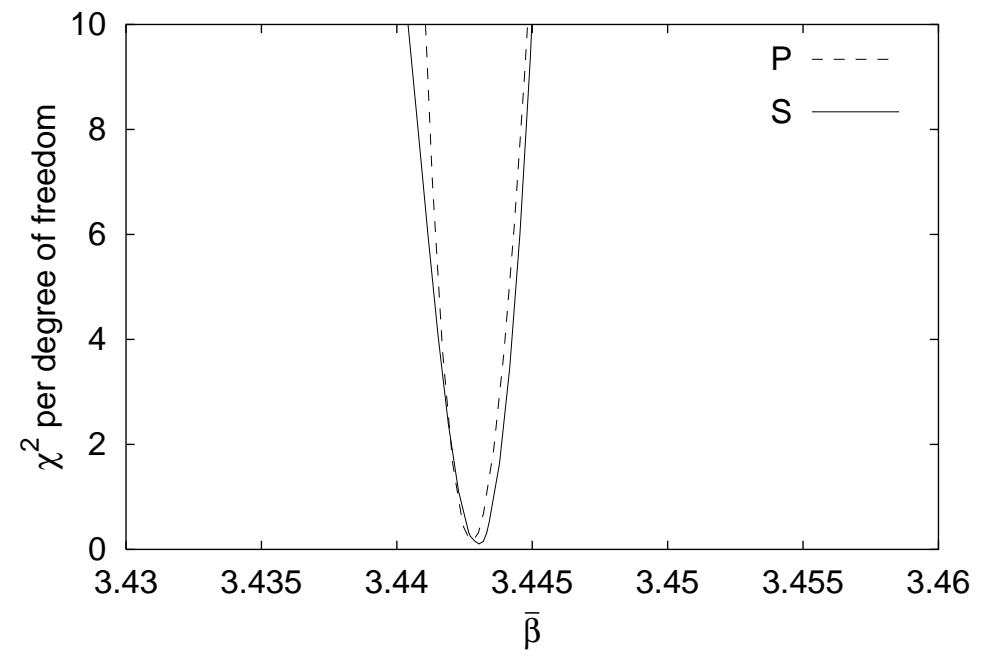

Figure 2: $\chi^{2}$ distributions for scaling fits of $P(\bar{\beta})$ and $S(\bar{\beta})$.

expectation value of the Polyakov loop gives $\bar{\beta}_{c}(L) \simeq 3.464$ as the critical point on $N_{s}^{2} \times 2$ lattices in the limit $N_{s} \rightarrow \infty$ [13], giving us an idea of the range to be studied.

A first scan for values $3.1 \leq \bar{\beta} \leq 3.5$ leads to the behaviour of $S(\bar{\beta})$ shown in Fig. 1. It is seen that $S(\bar{\beta})$ peaks slightly below $\bar{\beta}_{c}(L)$; with increasing $N_{s}$, the peak moves towards $\bar{\beta}_{c}(L)$ and the peak height increases.

In a second step, we carried out high-statistics simulations in a narrower range $3.410 \leq$ $\bar{\beta} \leq 3.457$ around the transition. In general, we performed between 30000 and 55000 measurements per $\bar{\beta}$ value, with the higher number taken in the region of the interval closest to the eventual critical point. To obtain the behaviour in the limit $N_{s} \rightarrow \infty$, we applied the density of states method (DSM) [14]. This method generates for each lattice size by interpolation further values of $P(\bar{\beta})$ and $S(\bar{\beta})$. The percolation critical point $\bar{\beta}_{c}(P)$ and the critical exponents are then determined through the $N_{s}$-dependence of the observables. At $\bar{\beta}_{c}(P)$, fits of the form $P(\bar{\beta}) \sim N_{s}^{-\beta / \nu}$ or $S(\bar{\beta}) \sim N_{s}^{\gamma / \nu}$ should lead to a 
minimal $\chi^{2}$ [15. Fig. 2 shows the $\chi^{2} /$ d.f. resulting from fits of $\log P$ and $\log S$ vs. $\log N_{s}$ at each value of $\bar{\beta}$ in the range $3.43 \leq \bar{\beta} \leq 3.46$. The two $\chi^{2}$ curves show pronounced minima with remarkable overlap.

In Table 1 we show the results for $95 \%$ confidence level, comparing the critical $\bar{\beta}_{c}(P)$ for Polyakov loop percolation to the $\bar{\beta}_{c}(L)$ from spontaneous symmetry breaking [13], and the percolation exponents to the Onsager values for the two-dimensional Ising model $(\beta=1 / 8, \gamma=7 / 4, \nu=1)$. The critical exponents are seen to be in excellent agreement. Since $\nu$ is determined by taking derivatives of the interpolated curves of $P(\bar{\beta})$ and $S(\bar{\beta})$ with respect to $\bar{\beta}$, the precision of its determination is reduced in comparison to that of $\beta / \nu$ and $\gamma / \nu$. The critical values of $\bar{\beta}$ are very close, but they do not overlap within errors. In view of the approximate solution for $\kappa(\bar{\beta})$ used here, small deviations are not unexpected. Monte Carlo renormalisation group techniques may well allow a more precise determination of the bond weight (12). [16].

\begin{tabular}{|c||c|c|c|c|}
\hline & $\bar{\beta}_{c}$ & $\beta / \nu$ & $\gamma / \nu$ & $\nu$ \\
\hline \hline Percolation & $3.443_{-0.001}^{+0.001}$ & $0.128_{-0.005}^{+0.003}$ & $1.752_{-0.008}^{+0.006}$ & $0.98_{-0.04}^{+0.07}$ \\
\hline Spont. Symm. Breaking & $3.464_{-0.016}^{+0.012}$ & 0.125 & 1.75 & 1.00 \\
\hline
\end{tabular}

Table 1: Critical parameters for $S U(2)$ lattice gauge theory with $N_{t}=2$.

We thus conclude that for the specific case considered here, two-dimensional $S U(2)$ lattice gauge theory with $N_{t}=2$, deconfinement can indeed be specified through Polyakov loop percolation. To make this more general, further work is necessary. The most crucial open problem is certainly the general form of $\kappa$ in Eq. (12). In particular, it is not clear why the general $S U(2)$ action should lead to a nearest-neighbour form, and how the 'temperature' $\kappa$ can be defined more generally, away from the strong coupling limit $N_{t}=2$. For the latter problem, the use of Monte Carlo renormalisation group techniques [17] may be of help. If and when these problems are solved, the corresponding percolation study has to be carried out for three space dimensions and for $S U(3)$ gauge theory as well, where the transition becomes first order.

Finally we return to the motivation mentioned at the beginning. For spin systems, percolation in coordinate space remains as critical phenomenon even in the presence of an external field [18], although this criticality is now not connected any more to singular behaviour of the partition function. In principle, this could provide the basis for a deconfinement order parameter in full QCD with dynamical quarks [19].

\section{Acknowledgements}

H.-W. Huang participated in the early stages of this work; we thank him very much for his helpful contributions. Furthermore, we would like to thank Ph. Blanchard, P. Białas, J. Engels, D. Gandolfo and F. Karsch for stimulating discussions. The financial support of the EU-Network ERBFMRX-CT97-0122 and the DFG Forschergruppe Ka 1198/4-1 is gratefully acknowledged. 


\section{References}

[1] L. D. McLerran and B. Svetitsky, Phys. Lett. 98 B (1981) 195.

[2] J. Kuti, J. Polónyi and K. Szlachányi, Phys. Lett. 98B (1981) 199.

[3] J. Polónyi and K. Szlachányi, Phys. Lett. 110B (1982) 395.

[4] B. Svetitsky and L. G. Yaffe, Nucl. Phys. B 210 [FS6] (1982) 423.

[5] J. Engels et al., Phys. Lett. B 365 (1996) 219.

[6] C. M. Fortuin and P. W. Kasteleyn, Physica 57 (1972) 536.

[7] R. J. Baxter, S. B. Kelland and F. Y. Wu, J. Phys. A 9 (1976) 397.

[8] A. Coniglio and W. Klein, J. Phys. A 13 (1980) 2775.

[9] R. H. Swendsen and J.-S. Wang, Phys. Rev. Lett. 58 (1987) 86.

[10] H. Meyer-Ortmanns, Z. Phys. 27 (1985) 553.

[11] U. Wolff, Phys. Rev. Lett. 62 (1989) 361.

[12] F. Green and F. Karsch, Nucl. Phys. B 238 (1984) 297.

[13] M. Teper, Phys. Lett. B 313 (1993) 417.

[14] M. Falconi et al., Phys. Lett. 108 B (1982) 331;

E. Marinari, Nucl. Phys. B 235 (1984) 123;

G. Bhanot et al., Phys. Lett. 183 B (1986) 331;

A. M. Ferrenberg and R. H. Swendsen, Phys. Rev. Lett. 61 (1988) 2635 and 63 (1989) 1195.

[15] J. Engels et al., Phys. Lett. B 365 (1996) 219.

[16] A. Goksch and M. Ogilvie, Phys. Rev. Lett. 54 (1985) 1772;

R. V. Gavai, A. Goksch and M. Ogilvie, Phys. Rev. Lett. 56 (1986) 815;

M. Okawa, Phys. Rev. Lett. 60 (1988) 1805.

[17] A. Gonzales-Arroyo and M. Okawa, Phys. Rev. D 35 (1987) 672:

M. Okawa, Phys. Rev. Lett. 60 (1988) 1805.

[18] J. Kertész, Physica A 161 (1989) 58.

[19] H. Satz, Nucl. Phys. A642 (1998) 130c. 